\title{
Overview of in vitro and in vivo doubled haploid technologies
}

Jose M. Seguí-Simarro $^{1 *}$, Nathanaël M.A. Jacquier ${ }^{2,3}$ and Thomas Widiez ${ }^{2}$

${ }^{1}$ Cell Biology Group - COMAV Institute, Universitat Politècnica de València, Valencia, Spain.

${ }^{2}$ Laboratoire Reproduction et Développement des Plantes, Univ Lyon, ENS de Lyon, UCB Lyon 1, CNRS, INRAE, F-69342, Lyon, France.

${ }^{3}$ Limagrain, Limagrain Field Seeds, Research Center, F-63360 Gerzat, France.

Running head: Overview of doubled haploid technologies

PMID: 34270023 DOI: 10.1007/978-1-0716-1315-3_1

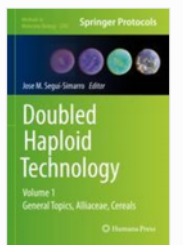

Doubled Haploid Technology. pp 3-22 | Cite as

Overview of In Vitro and In Vivo Doubled Haploid Technologies

Authors

Authors and affiliations

Jose M. Seguí-Simarro $\square$, Nathanaël M. A. Jacquier, Thomas Widiez 


\section{i. Chapter Title: Overview of in vitro and in vivo doubled haploid technologies}

\section{ii. Abstract}

Doubled haploids (DH) have become a powerful tool to assist in different basic research studies, and also in applied research. The principal (but not the only) and routine use of DH by breeding companies is to produce pure lines for hybrid seed production in different crop species. Several decades after the discovery of haploid inducer lines in maize and of anther culture as a method to produce haploid plants from pollen precursors, the biotechnological revolution of the last decades allowed to the development of a variety of approaches to pursue the goal of doubled haploid production. Now, it is possible to produce haploids and DHs in many different species because when a method does not work properly, there are several others to test. In this chapter, we overview the currently available approaches used to produce haploids and DHs by using methods based on in vitro culture, or involving the in vivo induction of haploid embryo development, or a combination of both.

iii. Key words: Androgenesis, Embryogenesis, Gynogenesis, Doubled haploid, Haploid, Haploid inducer, Tissue culture. 


\section{Introduction}

As opposed to animals, plants have a remarkable developmental plasticity. This is reflected in the totipotency of differentiated plant cells that can undergo a transition towards an undifferentiated, proliferative growth, forming callus masses, and/or switch towards other developmental programs, different from their original one. This way, new plants from individual cells can be regenerated through organogenesis, promoting the successive formation of all their organs. Alternatively, the expression of the embryogenic program (embryogenesis) can be promoted, thereby transforming plant cells into functional zygote-like structures which will become embryos and eventually plants. Virtually any plant cell type can be used, if optimal conditions (experimental treatments) are found to promote organogenesis and/or embryogenesis, which opens up a wealth of biotechnological possibilities. Among these cell types, the cells of the germ line (gametes or their precursors) are one of the most interesting from a biotechnological perspective, because they contain half of the chromosomes found in somatic cells, and are thus haploid.

Haploid generally refers to the product of meiosis, which, in a cell with a complete set of chromosomes (2n) leads to gametes with only half of this set (n). By convention " $n$ " and "2n" refer to the gametic and sporophytic chromosome numbers, respectively, and " $\mathrm{x}$ " denotes the number of sets of chromosomes (Figure 1A). Depending on the ploidy level of the organism, a haploid tissue/organism may have more than one copy of each homologous chromosome (Figure 1A). However, haploidy is not restricted to gametes in the plant kingdom. The life cycle of bryophytes (Figure 1B), for instance, relies mostly on the gametophyte which is the haploid phase with a short-lived 2n sporophyte dependent on the n gametophyte [1]. During evolution, plants progressively reduced their haploid phase. Haploid tissues (Figure 1B) were restricted spatially and temporally and became dependent for development and nutrition on $2 \mathrm{n}$ sporophytic tissues [1]. The extended life cycle proportion of the sporophyte provided 
evolutionary advantages [2]. For instance, deleterious alleles, hence characters, might be hidden by the dominant ones held on homologous chromosomes.

From haploid cells, the artificial generation of new haploid individuals, which may become doubled haploid (DH), can be induced. To produce a $\mathrm{DH}$, haploid cells (genetically unstable in essence) may undergo duplication of their genome at any time during their proliferation, becoming diploid with no need for additional treatments. Genome duplication is typically achieved by nuclear fusion after incomplete cytokinesis [3]. Since the second chromosome set is identical to the original one, they are true DHs, $100 \%$ homozygous. This is commonly known as spontaneous genome doubling, in the sense that nothing is done to specifically promote genome doubling, aside of the treatments applied to induce haploid embryogenesis itself, which may also have an indirect side effect on genome doubling. This is how DHs are produced in many instances, where the percentage of unaided genome doubling is high enough to make the direct disposal of haploid individuals cost-effective, keeping only those that effectively doubled their genome by themselves. However, different genetic backgrounds are differently prone to undergo such phenomenon without the application of additional treatments for genome doubling. In other cases, this percentage is very low, which makes mandatory the implementation in the $\mathrm{DH}$ protocol of such a treatment to stimulate doubling of haploid embryos. Among them, the most effective and widely used is by far the application of colchicine, an antimitotic drug. Chapter 9 of this book compiles the principal methods used for chromosome doubling applied to $\mathrm{DH}$ production.

Haploids themselves are useful experimental systems to study, for example, the effects of recessive mutations, since their phenotypes are not masked by dominant alleles. However, their principal utility is to serve as the starting point to obtain DH lines. DHs are individuals whose diploid genome comes from a haploid set of chromosomes that has been duplicated, so that all their loci contain the same alleles. They are $100 \%$ homozygous individuals, and represent a 
valuable tool for basic and applied research, including breeding programs, where they are often used as pure lines for hybrid seed production. Chapter 2 of this book compiles the principal applications of DHs in both applied research and plant breeding.

Induced haploid embryos/plantlets have only one of the two copies of the genome of the male or female donor plant they come from. Due to this, it is common to refer to them as "male/paternal” or “female/maternal” haploids, respectively. Theoretically, haploid cells can only have two origins. They must come:

- Either from male haploid nuclei derived from meiosis of microspore mother cells. The origin of these haploid nuclei would potentially include meiotic products, still within the tetrad, not released as microspores, individual microspores once released from the tetrad, or any of the different cells of the pollen grain, the vegetative cell, the generative cell, or the sperm cells (the male gametes) produced from the latter.

- Or from female haploid nuclei derived from meiosis of megaspore mother cells. The origin of these haploid nuclei would potentially include the functional megaspore, either included or released from the meiocyte, and any of the haploid cells of the embryo sac: synergid cells, antipodal cells, and the egg cell, the female gamete.

In practice, only some of these haploid cells have been demonstrated to produce haploid/DH plants. They include microspores at different stages, young pollen grains and egg cells. However, we cannot rule out the possibility of other haploid cells being able to be induced [4]. These cells can be induced to produce haploid and/or DH plants by means of different techniques, which exploit a series of phenomena observed to occur in plants under natural or experimental conditions. These techniques can be grouped into two main categories, in vitro and in vivo methods, depending on whether they include exclusively in vitro procedures, or there are stages of in vivo development of haploid/DH individuals (Figure 2). In this chapter, we will review the known in vitro and in vivo methods to produce $\mathrm{DH}$ individuals. 


\section{In vitro methods}

The most widely extended approaches to obtain DHs have traditionally been based on the use of haploid cells of male and female origin to induce their development as haploid embryos by the application of different stresses in vitro and their subsequent in vitro culture. They are the so-called in vitro approaches (Figure 2). The production of haploid/DH plants from male haploid cells is commonly known as induction of in vitro androgenesis, whereas production of haploid/DH plants from female haploid cells is commonly known as induction of in vitro gynogenesis. The different strategies have in common the blockage of the normal development of these cells, whose natural fate is the production of functional gametes or accessory cells, and their in vitro reprogramming towards a different developmental fate, which is to become embryos without fertilization. This way, haploid and/or DH individuals can be produced in vitro.

In the last six decades, haploid cells of both male and female origins have been used to produce DHs in vitro, although with different success rates. In general, the haploid cells where in vitro haploid/DH induction has been most successful are male microspores and female egg cells. In particular, in vitro production of androgenic DHs has been more successful than production of gynogenic DHs due to several reasons:

- First, male haploid cells are by far more abundant. In a given hermaphrodite flower, thousands of microspores or pollen grains are present in each of the several anthers of a flower, whereas there is only one functional megaspore, which gives rise to six haploid cells per embryo sac, including the egg cell, per ovule. Different species may have different number of ovules per flower, and in some cases there may be up to hundreds of ovules, but 
this number will never be comparable to the enormous number of microspores/pollens produced by the same flower.

- Second, female haploid cells are confined in the interior of ovules, surrounded by layers of nucellar and tegument tissues, all this being included within the ovary. This confines the newly formed haploid embryos within a very small space that does not enlarge in parallel to the embryo, because the ovule and ovary do not receive the necessary developmental cues to develop in parallel to the haploid embryo. On the other hand, androgenic embryos will only have to overcome the barrier imposed by anther walls, which are naturally programmed to dehisce and open along the dehiscence lines or pores. Thus, gynogenic embryos will have it more difficult to emerge from the surrounding tissues, which will account for the reduced rates of success.

- Third, in nearly all cases, the individuals regenerated from female haploid cells remain haploid, which makes always mandatory a step of genome doubling.

Both androgenic and gynogenic embryos may be produced through different in vitro experimental approaches, as explained next.

\subsection{Methods based on in vitro androgenesis}

Methods based on androgenesis exploit the possibility of switching the developmental fate of pollen precursors towards embryogenesis. The most used pollen precursors are, by far, microspores/young pollen grains. Microspore embryogenesis (also known as pollen embryogenesis) is by far the most used and efficient way to produce DHs in vitro. This experimental pathway was first discovered by Guha and Maheswari in 1964 [5], while working with in vitro cultured anthers of Datura innoxia. Later on, many different research groups have reproduced their findings in many other species and genera, making this experimental 
phenomenon a powerful and widespread tool to produce DHs. However, not all the species respond equally to the induction of this process. Some species, considered models for the study of this phenomenon, respond fairly well. This is the case of certain lines of rapeseed (Brassica napus), tobacco (Nicotiana tabacum), or barley (Hordeum vulgare). Others, considered recalcitrant, present a low or very low response, and in other cases, a protocol to efficiently induce this process is still pending to be developed, as for scientifically or agronomically important species such as Arabidopsis thaliana or tomato (Solanum lycopersicum), respectively. Many other species are in between these two extreme situations, being possible to induce microspore embryogenesis, but with yet improvable protocols. Woody species are good examples of materials where some success has been achieved, but there is still a large room for improvement (reviewed in [6-8]). Chapter 3 of this book includes a list of species where production of haploids/DHs has been assessed by this approach.

Even within a species, there will be varieties, lines and even individuals that respond differently. This strong influence of the genotype, together with the fact that this trait is transmitted across generations and segregates in the hybrids offspring $[9,10]$, indicates that it is under genetic control [11-17]. Furthermore, it was proposed that, at least for Brassica napus, the embryogenic competence of microspores is controlled by two loci with additive effects [10]. The gene or genes involved, however, remain to be elucidated.

In microspore embryogenesis, androgenic haploids/DHs are typically produced upon deviation of microspores towards embryogenesis or callus formation. However, not all species can be induced by isolation and culture of microspores at the same stage (Figure 3). Indeed, for few species it has been shown that male-derived haploid and DH plants can be regenerated from meiocyte-derived callus [18-20]. This is a very exceptional route, much less frequent and studied than microspore embryogenesis. Under certain circumstances, meiocytes at late meiosis stages, always after recombination but before the release of the four individual microspores, 
can be induced to proliferation (Figure 3, Route 1), forming undifferentiated haploid callus masses where haploid/DHs can be regenerated from. Meiocyte-derived callogenesis has been documented in Arabidopsis thaliana, Vitis vinifera Digitalis purpurea and Solanum Lycopersicum [21-32]. In addition to its rare occurrence and the scarcity of studies, the practical use of this alternative is hampered by a very low frequency of cases, since many of the regenerants produced come from the fusion of two meiotic haploid products separated by defective, incomplete, or absent cell walls (Figure 3, Route 1) [25]. This will give rise to a majority of non-DH, useless plants that must be identified as such and then discarded. Altogether, these limitations make that in practice, this in vitro alternative is not used.

For some species, mostly cereals, the early stages of microspore development have been described as the only stage where embryogenesis can be induced [33], However, the majority of works point to the fact that the inducible developmental window revolves around the first pollen mitosis (Figure 3, Route 2), which means that vacuolated microspores but also young, just divided pollen grains would also be inducible (reviewed in [33,19,34]. The identification and isolation of these stages is essential for the success of this process, since in the vast majority of species, they are the most sensitive to the induction treatments [35]. Beyond these stages, induction has only exceptionally been reported [36].

To be induced to embryogenesis, microspores/pollens must be stressed. The need for application of physicochemical stress treatments seems common to all inducible species. The variety of responses, depending principally on the genotype but also on the developmental stage of the microspore/pollen, makes that each species has its own specific inductive treatments to trigger the developmental switch. Some of these stresses (heat, cold or starvation) are common to many species, whereas others need more specific stressors or combinations of them [37]. As a rule of thumb, the more recalcitrant a species is, the more combined and more intense stresses are needed. Typically, induction of microspore embryogenesis produces microspore-derived 
embryos. However, there are some species where microspores proliferate in an undifferentiated manner, producing callus masses (Figure 3, Route 2; reviewed in [19]). In other cases, embryos cannot progress as such and proliferate as callus masses [38-40]. As pointed out previously [19], this may be due the absence of developmental regulation in this type of in vitro embryogenesis, devoid of endosperm and other seed tissues that may interact with the embryo. When suboptimal culture conditions do not compensate for this, abnormal embryos or even calli may arise.

The process of microspore embryogenesis and the different factors, mentioned above, that influence its success, may be implemented in practice using two in vitro approaches: anther culture and isolated microspore culture.

\subsubsection{Anther culture}

Anther culture is the most universal method to produce DHs. It is technically simple, consisting basically of the following steps: (1) flower bud collection, (2) isolation of anthers from flower buds, (3) inoculation and in vitro culture in agar-based culture medium, (4) isolation of embryos, (5) regeneration of plants, and (5) analysis of regenerants. Few weeks (months in many cases) after, microspore-derived embryos may be seen to emerge from anther walls, in parallel to the degradation and necrosis of these walls. In general, a given anther under optimal culture conditions may give rise to several tens of microspore-derived embryos during several months of culture. The presence of these walls (the tapetum principally) during the first stages of anther culture may protect and help microspores to undergo the first stages of haploid development, in a way similar to how they assist normal microspore development in vivo. Perhaps, this is the reason why anther culture works in many different species, including those where other DH methods do not work (See chapter 3). 
However, anther cultures are not devoid of limitations. Perhaps, the main limitation comes from the fact that microspores are cultured together with anther walls. Anther walls (the tapetal layer mostly) may secrete molecules that may protect microspores or promote their growth, but it may also secrete inhibitory or even toxic compounds, as is the case of necrosing anther tissues. In any case, this secretory effect is uncontrollable in essence, and makes difficult a strict control of culture conditions. Moreover, when exposed to growth regulators, these walls are able to proliferate in vitro, producing calli. Indeed, some parts of the anther, such as the filament insertion, are especially prone to form calli when in vitro cultured. Therefore, we cannot rule out the possibility of occurrence of somatic embryos (very rare but possible) and calli (much more frequent) from anther walls. This implies that for every single plant confirmed as diploid (2C DNA content) by flow cytometry, we should check its origin. For this, the most reliable approach is the use of molecular markers previously confirmed as heterozygous in the donor plants used (see Chapter 10). However, for very well-known cultivars, where repeated analyses have shown that no somatic embryos are produced, this step may be skipped simply by discarding all calli produced and using only embryos.

\subsubsection{Isolated microspore culture}

Microspore embryogenesis can also be induced using microspore isolated from anthers. This alternative is more complex than anther culture because a step of microspore isolation and inoculation into liquid medium must be implemented to the protocol. In addition, the absence of anther tissues makes that proper microspore growth and development will exclusively depend on medium composition. Thus, in order to develop an efficient protocol for microspore culture, medium composition must include all the elements needed by microspores and must be adjusted to the particularities of the microspores of each species. The use of liquid culture 
media increase the risk of contamination compared with anther cultures, which use to be performed in agar-based, semisolid media.

Together with these limitations, isolated microspore cultures have also some advantages that, in some cases, largely surpass the limitations. Microspore cultures avoid the incontrollable contribution of anther walls, and the potential toxicity of anther wall degradation products. In addition, all nutrients and active compounds of the medium are easily available by microspores, suspended in the liquid medium instead of confined within the anther locule. These features are likely behind the fact that microspore cultures are notably faster than anther cultures. Indeed, in some model species where protocols are optimized, few weeks are needed produce hundreds of embryos from the microspores inoculated in a single dish [41], see Chapter 18. In addition to this, microspore cultures avoid the routine, time-consuming procedure of checking the haploid origin of all the diploid plants obtained. Since microspores are isolated from all other anther tissues and only microspores are inoculated into the dishes, the only possible origin for embryos or calli must be the microspores. In other words, all diploid regenerants obtained will be DHs. All these advantages considered, isolated microspore culture is the method of choice in those materials where efficient protocols are well established.

\subsection{1 Methods based on in vitro gynogenesis}

Gynogenesis would exploit the ability of egg cells to develop in the embryo sac as a haploid zygote without fertilization (Figure 4, Route 4). This alternative to the normal development of the megagametophyte was first described in vitro in 1976 by San et al. [42]. It would therefore be a form of female haploid parthenogenesis (from the Greek words parthenos, meaning “virgin” and genesis, meaning “origin”. In some species, the gynogenic embryo is believed to originate from antipodal or synergid cells, but in the vast majority of cases the gynogenic 
embryo is derived from the egg cell (reviewed in [4]). Gynogenic embryos are mostly haploid, which implies that in order to obtain the desired double haploid, the application of additional treatments for chromosome duplication should be considered in nearly all cases. As in the case of androgenesis, colchicine is the most effective and therefore the most widely used antimitotic (Chapter 9).

The success of gynogenesis induction is influenced by many different factors, including the developmental stage of the embryo sac and the in vitro culture conditions. However, the genotype is the most important, even more than for microspore embryogenesis. In fact, this is the most limiting factor for the practical application of this technique, since there are very few responsive genotypes, much less than those that respond to microspore embryogenesis. Other limitations include a low efficiency, much lower than microspore embryogenesis (there are much less egg cells than microspores in a flower), a very low rate of spontaneous duplication of the genome, and low levels of embryo regeneration, perhaps due to the instability of haploid genomes, prone to chromosomal alterations.

All these limitations make in vitro gynogenesis-based approaches a secondary alternative, used in a reduced range of species where other in vitro approaches (microspore embryogenesis) have proven ineffective. Chapter 3 of this book includes a list of species where production of haploids/DHs has assessed by this approach. Among them, the model species for the study of this process is onion (Allium cepa) [43,44] See Chapter 13.

From a methodological point of view, this technique is implemented by in vitro culture of ovules [45,46], ovaries [47] or even full immature flowers [43], not yet open and therefore unpollinated, until the embryo sac matures and the gynogenic embryo develops. In some species, mere in vitro culture seems not enough, and an "extra" factor must be applied to trigger the process. Examples of this factor include include pollination before ovary excision and in vitro culture [48], in vitro pollination with mentor pollen from other species [49], with pollen 
irradiated with gamma or X rays to inactivate its fertilization capability [50] or with triploid pollen, still able to germinate and stimulate the egg cell, but not to fertilize. In vitro pollination can be done at the apical part of the stigma of entire pistils isolated from the flower and cultured in vitro [51]. After several months, gynogenic embryos will be visible. Alternatively, pollen may be applied by placental pollination, which implies isolating the ovules from the ovary, but maintaining a fragment of the placenta to help the viability of the egg cell. In many cases of gynogenesis in cucurbits and fruit trees, treated pollen is applied directly in situ, on the emasculated flower in the plant [52-56]. Then, seeds or haploid embryos are rescued and in vitro cultured (see also Section 3.2). These approaches would be half way between the in vitro approaches and the in vivo approaches described next (Figure 2).

\section{In vivo approaches}

Alternatives to the in vitro approaches to generate haploid plantlets are in vivo approaches (Figure 2). Overall, in vivo methods are less numerous as compared to in vitro methods and thus less plants/crops are concerned. In vivo methods look attractive because they appear to be "simpler", since the plant is mainly doing the job instead of labor intensive in vitro work. Nevertheless, improvement and optimization are usually needed between the discovery of an in vivo induction system and its application in breeding scale. Indeed, long standing in vivo haploid induction methods exist $[57,33,58]$ and some have been improved and are currently used on a routine basis in breeding programs. Examples of these methods include the use of maize haploid inducer lines (see Chapter 6) or the so-called wide crosses. More recently (10 years ago), new in vivo methods have been discovered [59], and are currently tested for translation to crops (e.g. centromere engineering, see Chapter 7). In vivo haploid induction methods could be divided into two main broad categories: (1) the "wide-crosses" and (2) the intra-specific crosses (Figure 2). 


\subsection{Haploidization by wide-crosses}

Wide crosses consist to force crosses between species spanning wide taxonomic boundaries. It could thus involve inter-generic or inter-specific pollinations, and often concern crosses between a cultivated crop and a wild-relative species. Wide crosses are also named wide hybridization, and imply to overcome pre-fertilization and post-fertilization barriers [60]. They have been reported more frequently in monocotyledonous species as compared to dicotyledonous [57].

Two different outcomes, both useful for breeders, need to be distinguished from these wide crosses. Firstly, in case of successful hybridization, which could be helped by embryo rescue or other techniques [60], the production of hybrid embryos is a starting material in order to introduce agronomic traits of interest (disease resistance, stress tolerance...etc.) across species. Secondly, it could be used for the production of haploid embryos. Due to the unstable nature of hybrid embryos generated by joining two different genetic materials, chromosome elimination from one parent occurs during early embryogenesis in some “wide-crosses” [57,61,58,19]. Although the paternal chromosomes are eliminated in most of the cases to give rise to maternal haploid embryos (Figure 4, route 5), some rare cases were reported in which the paternal genome remains, being the maternal genome eliminated (Figure 3, route 3) (reviewed in [19]). A pioneering discovery in haploidization by wide-crosses was the Bulbosum method (Chapter 25), which has been well studied and is now widely used in barley breeding [57,62]. Cross of cultivated barley (Hordeum vulgare) using pollen from its wild relative Hordeum bulbosum leads to the production of $H$. vulgare haploid embryos $[63,64]$. The success of barley haploid embryo production thanks to wide crosses was then extended to other species, especially using maize pollen which appears to display low-intensity fertilization barriers [65,57]. For example, wheat and triticale haploid embryos are currently obtained in some plant breeding programs by 
the following crosses: wheat $\times$ maize, and triticale $\times$ maize respectively $[66,57,67]$. Overall, wide crosses are limited to some crops at breeding scale, but once they are well established methods, they have the advantage to be effective across a wide range of genotypes, as opposed to the in vitro approaches, which are highly genotype-dependent within a given species. Nevertheless, a limitation of the wide crosses method is that it is not fully in vivo (Figure 2), since it necessitates to include a stage of in vitro tissue culture to prevent embryo abortion. Indeed, successful seed development relies on the correct development of the two fertilization products: the embryo and the endosperm. The endosperm tightly interacts, both physically and chemically, with the embryo and it is thus of vital importance to sustain embryo development [68-70]. In haploid embryo-producing wide crosses, the endosperm fails to develop properly, probably due to selective parental chromosome elimination occurring in this tissue as well, and consequently haploid embryos must be rescued by in vitro culture.

\subsection{Haploidization by intra-specific crosses}

Two main methods could be differentiated in order to induce haploid embryos via intra-specific crosses: (1) pollination with treated pollen, and (2) the use of haploid inducer lines. While methods based on treated pollen usually necessitate haploid embryo rescue due to early seed abortion [71,33], the haploid inducer lines present the advantage to be fully in planta because the output is the production of viable seeds containing haploid embryos (Figure 2) [72,73]. Depending on the methods and species considered, haploidization by intra-specific crosses could produce two different kinds of haploid embryos: maternal haploid embryos with the cytoplasm and nuclear genome from the female parent (Figure 4, route 4 and/or route 5), and paternal haploid embryos, having the cytoplasm of the egg cell (maternal) but the nuclear genome from the male parent (Figure 3, route 3). In the latter case, male haploid embryos produced by intra-specific crosses might be additionally useful for other biotechnological 
purposes beyond conventional production of DH pure lines. Indeed, since mitochondrial defects are behind cytoplasmic male sterility (CMS), CMS is maternally inherited through the cytoplasm $[74,75]$. CMS is a valuable tool in hybrid seed production, since it avoids the timeconsuming process of emasculation to prevent self-pollination [76]. This trait is traditionally transferred from one germplasm to another through multiple rounds of backcrossing. Obtaining a nuclear male genome within a "maternal” cytoplasm in just a single cross reduces CMS conversion to just one step, thus accelerating hybrid seed production.

Pollinations with treated pollen induce maternal haploid embryos (Figure 4, routes 4, 5). This method consists in the treatment of pollen, prior to pollination, with physical or chemical agents, irradiation being the most used treatment [71,33]. Although haploidization via pollen treatments has been reported in more than 15 species [71,33], it works ineffectively (low haploid induction rate). Thus, this method is used in plant breeding only when no alternative efficient methods are available, for example in melon and cucumber [77]. Haploid inducer lines could be seen as an exception since they exist in few species only [58,78,73]. Moreover, haploid inducer lines are routinely used in plant breeding in maize (Chapter 6) and potato [79]. Intensive researches are currently being done to extend the CENH3-based inducer line to others crops (Chapter 7). In addition, the identification of the causal genes leading to embryo haploid induction in maize allowed for the translation of this trait to two other crops: wheat and rice [80-82]. Lastly, recent patents and publications reported on the identification of sorghum haploid inducer lines [83,84].

In maize, two different types of haploid inducer lines have been reported, which are able to produce either maternal or paternal haploid embryos (Figure 4, route 5 and Figure 3, route 3, respectively) $[85,86]$. Chapter 6 details the properties and uses of these two maize haploid inducer lines. These two lines gained their haploid induction phenotypes due to mutations in genes involved in male and female gametophyte development, and in double fertilization. 
In potato (Solanum tuberosum L.), the haploid induction system relies on a cross between a diploid male haploid inducer line (S. tuberosum Andigenum group, previously referred to as cultivar S. Phureja) with a tetraploid cultivated potato of interest used as female parent (Figure 4 , route 5 [79,87-89]. It thus refers to an interploidy cross (4x potato $\times 2 x$ haploid inducer line), and the haploid embryos found in some of the viable seeds of this cross are commonly called dihaploids to indicate that they contain two sets of chromosomes (from maternal origin). Such dihaploid plants are not homozygous, but allow breeders to work at the diploid level for simpler genetic analysis/mapping, or for introgression of valuable traits from the wild species. Since wild species are mostly diploid, they could be then crossed with the di-haploid by inter-specific hybridization $[87,88]$.

The CENH3-based haploid inducer lines originate from the manipulation of the centromeric histone protein CENH3 in Arabidopsis thaliana [59]: it was reported that the genome of the parental having the engineered CENH3 is eliminated after the cross with wild-type plants with intact CENH3, creating haploid inducer lines (Chapter 7 and for recent reviews: $[73,61,58,90]$. These CENH3-based haploid inducer lines are thus able to induce either maternal or paternal haploid embryos (Figure 4, route 5 and Figure 3, route 3), although they seem more efficient in producing paternal haploid embryos, in Arabidopsis at least [59]. Although CENH3 is conserved across plant species, efficient translation of this haploid induction method to crops remain to be achieved, since very low haploid induction rates have been observed so far in crops $[78,73,58]$.

To sum-up, haploidization by intra-specific crosses is attractive since haploid embryos are formed within a viable seed, but remain limited to few crops. Once haploid inducer lines have been reported or created, the main limitations/constraints for the use haploid inducer lines in breeding programs are the need for a relatively high haploid induction rate, and the existence of a system to identify the seeds having haploid embryos among the seeds having diploid 
embryos . The history of the maize male haploid inducer line exemplifies the important need of research and development to achieve a "good” haploidization method by intra-specific crosses: indeed more than 20 years separate the discovery of the first maize haploid inducer line by Ed Coe [86] from its use in maize breeding programs [91,92]. The improvements of both haploid induction rate (from $~ 2-3 \%$ to $~ 10 \%$ ) and color markers to accurately identify haploid embryos were some of the key steps in the successful use of maize haploid inducer lines in breeding programs (Chapter 6). Recently, the knowledge gained in the mode of action behind the maize in vivo haploid induction system [73,72], allowed for the successful translation of this feature to two new crops: rice and wheat [80-82].

\section{Concluding remarks}

In this chapter we have revised the principal approaches currently available to produce haploids and DHs (Figure 2) for different purposes, principally focused on the rapid generation of pure lines to accelerate hybrid seed production or CMS conversion and to simplify breeding programs by producing di-haploids, as for potato. These approaches imply the use of methods exclusively based on in vitro culture, in vivo induction of haploid development, or a combination of them to induce haploid embryos in vivo and then rescue them in vitro. Practical examples of the application of these methods to particular species or varieties are presented throughout the book. The choice of the best performing approach will depend on the species used, and to what extent these methods have been developed and adapted to this species. Together, these approaches illustrate how a given goal can be accomplished by different biotechnological means, and are a good example of the power of combining different biotechnological for solving specific applied problems of industry and in general, of society. 


\section{Acknowledgments}

We thank Luca Comai for the helpful discussions on potato haploid inducer lines. This work was supported by grant AGL2017-88135-R to JMSS from MINECO jointly funded by FEDER. T.W was supported by the ANR grant (ANR-19-CE20-0012), and by "pack ambition recherche” from the Région Auvergne-Rhone-Alpes (“HD-INNOV”). N.M.A.J was supported by CIFRE PhD fellowship from ANRT funding agency (grant no. 2019/0771).

\section{References}

1. Li W, Ma H (2002) Gametophyte development. Curr Biol 12 (21):R718-R721. doi:10.1016/s0960-9822(02)01245-9

2. Bennici A (2008) Origin and early evolution of land plants. Communicative \& Integrative Biology 1 (2):212-218. doi:10.4161/cib.1.2.6987

3. Seguí-Simarro JM, Nuez F (2008) Pathways to doubled haploidy: chromosome doubling during androgenesis. Cytogenet Genome Res 120 (3-4):358-369. doi:10.1159/000121085

4. Bohanec B (2009) Doubled Haploids via Gynogenesis. In: Touraev AF, BP Jain, SM (ed) Advances in Haploid Production in Higher Plants. Springer, pp 35-46

5. Guha S, Maheshwari SC (1964) In vitro production of embryos from anthers of Datura. Nature 204:497

6. Srivastava P, Chaturvedi R (2008) In vitro androgenesis in tree species: An update and prospect for further research. Biotechnol Adv 26 (5):482-491. doi:10.1016/j.biotechadv.2008.05.006

7. Andersen SB (2005) Haploids in the improvement of woody species. In: Palmer CE, Keller WA, Kasha KJ (eds) Haploids in crop improvement II, vol 56. Biotechnology in agriculture and forestry. Springer-Verlag, Berlin Heidelberg, pp 243-257

8. Germanà MA (2009) Haploids and doubled haploids in fruit trees. In: Touraev A, Forster BP, Jain SM (eds) Advances in haploid production in higher plants. Springer, Dordrecht, Netherlands, pp 241-263

9. Rivas-Sendra A, Campos-Vega M, Calabuig-Serna A, Seguí-Simarro JM (2017) Development and characterization of an eggplant (Solanum melongena) doubled haploid population and a doubled haploid line with high androgenic response. Euphytica 213 (4):89. doi:10.1007/s10681-017-1879-3

10. Zhang FL, Takahata Y (2001) Inheritance of microspore embryogenic ability in Brassica crops. Theor Appl Genet 103 (2-3):254-258

11. Barret P, Brinkman M, Dufour P, Murigneux A, Beckert M (2004) Identification of candidate genes for in vitro androgenesis induction in maize. Theor Appl Genet 109 (8):1660-1668

12. Kitashiba H, Taguchi K, Kaneko I, Inaba K, Yokoi S, Takahata Y, Nishio T (2016) Identification of loci associated with embryo yield in microspore culture of Brassica rapa by segregation distortion analysis. Plant Cell Rep:1-8. doi:10.1007/s00299-016-2029-4 
13. Malik MR, Wang F, Dirpaul J, Zhou N, Hammerlindl J, Keller W, Abrams SR, Ferrie AMR, Krochko JE (2008) Isolation of an embryogenic line from non-embryogenic Brassica napus cv. Westar through microspore embryogenesis. J Exp Bot 59 (10 ):2857-2873. doi:10.1093/jxb/ern 149

14. Beckert M (1998) Genetic analysis of in vitro androgenetic response in maize. In: Chupeau Y, Caboche M, Henry Y (eds) Androgenesis and haploid plants. Springer-Verlag, Berlin, Heidelberg, pp 24-37

15. Rudolf K, Bohanec B, Hansen M (1999) Microspore culture of white cabbage, Brassica oleracea var. capitata L.: Genetic improvement of non-responsive cultivars and effect of genome doubling agents. Plant Breed 118 (3):237-241

16. Yamagishi M, Otani M, Higashi M, Fukuta Y, Fukui K, Shimada T (1998) Chromosomal regions controlling anther culturability in rice (Oryza sativa L.). Euphytica 103 (2):227234. doi:10.1023/a:1018328708322

17. Zhang FL, Aoki S, Takahata Y (2003) RAPD markers linked to microspore embryogenic ability in Brassica crops. Euphytica 131 (2):207-213

18. Seguí-Simarro JM, Corral-Martínez P, Parra-Vega V, González-García B (2011) Androgenesis in recalcitrant solanaceous crops. Plant Cell Rep 30 (5):765-778. doi:10.1007/s00299-010-0984-8

19. Seguí-Simarro JM (2010) Androgenesis revisited. Bot Rev 76 (3):377-404. doi:10.1007/s12229-010-9056-6

20. Seguí-Simarro JM (2016) Androgenesis in solanaceae. In: Germanà MA, Lambardi M (eds) In vitro embryogenesis, vol 1359. Methods in Molecular Biology. Springer Science + Business Media, New York, pp 209-244. doi:10.1007/978-1-4939-3061-6_9

21. Gresshoff PM, Doy CH (1974) Derivation of a haploid cell line from Vitis vinifera and importance of stage of meiotic development of anthers for haploid culture of this and other genera. Z Pflanzenphysiol 73 (2):132-141

22. Gresshoff PM, Doy CH (1972) Haploid Arabidopsis thaliana callus and plants from anther culture. Australian Journal of Biological Sciences 25 (2):259

23. Corduan G, Spix C (1975) Haploid callus and regeneration of plants from anthers of Digitalis purpurea L. Planta 124 (1):1-11

24. Corral-Martínez P, Nuez F, Seguí-Simarro JM (2011) Genetic, quantitative and microscopic evidence for fusion of haploid nuclei and growth of somatic calli in cultured $\mathrm{ms}^{3} \mathrm{O}^{35}$ tomato anthers. Euphytica 178 (2):215-228. doi:10.1007/s10681-010-0303-z

25. Seguí-Simarro JM, Nuez F (2007) Embryogenesis induction, callogenesis, and plant regeneration by in vitro culture of tomato isolated microspores and whole anthers. J Exp Bot 58 (5):1119-1132

26. Bal U, Abak K (2007) Haploidy in tomato (Lycopersicon esculentum Mill.): a critical review. Euphytica 158 (1-2):1-9

27. Seguí-Simarro JM, Nuez F (2005) Meiotic metaphase I to telophase II is the most responsive stage of microspore development for induction of androgenesis in tomato (Solanum lycopersicum). Acta Physiol Plant 27 (4B):675-685

28. Zagorska NA, Shtereva LA, Kruleva MM, Sotirova VG, Baralieva DL, Dimitrov BD (2004) Induced androgenesis in tomato (Lycopersicon esculentum Mill.). III. Characterization of the regenerants. Plant Cell Rep 22 (7):449-456

29. Zagorska NA, Shtereva A, Dimitrov BD, Kruleva MM (1998) Induced androgenesis in tomato (Lycopersicon esculentum Mill.) - I. Influence of genotype on androgenetic ability. Plant Cell Rep 17 (12):968-973

30. Shtereva LA, Zagorska NA, Dimitrov BD, Kruleva MM, Oanh HK (1998) Induced androgenesis in tomato (Lycopersicon esculentum Mill). II. Factors affecting induction of androgenesis. Plant Cell Rep 18 (3-4):312-317 
31. Sharp WR, Raskin RS, Sommer HW (1972) The use of nurse culture in the development of haploid clones in tomato. Planta 104 (4):357-361

32. Gresshoff PM, Doy CH (1972) Development and differentiation of haploid Lycopersicon esculentum (tomato). Planta 107 (2):161-170

33. Dunwell JM (2010) Haploids in flowering plants: origins and exploitation. Plant Biotechnol J 8 (4):377-424. doi:10.1111/j.1467-7652.2009.00498.x

34. Germanà MA (2011) Anther culture for haploid and doubled haploid production. Plant Cell Tissue Organ Cult 104 (3):283-300. doi:10.1007/s11240-010-9852-z

35. Touraev A, Pfosser M, Heberle-Bors E (2001) The microspore: A haploid multipurpose cell. Adv Bot Res 35:53-109

36. Binarova P, Hause G, Cenklova V, Cordewener JHG, van Lookeren-Campagne MM (1997) A short severe heat shock is required to induce embryogenesis in late bicellular pollen of Brassica napus L. Sex Plant Reprod 10 (4):200-208

37. Shariatpanahi ME, Bal U, Heberle-Bors E, Touraev A (2006) Stresses applied for the reprogramming of plant microspores towards in vitro embryogenesis. Physiol Plant 127 (4):519-534

38. Rivas-Sendra A, Corral-Martínez P, Camacho-Fernández C, Seguí-Simarro JM (2015) Improved regeneration of eggplant doubled haploids from microspore-derived calli through organogenesis. Plant Cell Tissue Organ Cult 122 (3):759-765. doi:10.1007/s11240-015-0791-6

39. Corral-Martínez P, Seguí-Simarro JM (2014) Refining the method for eggplant microspore culture: effect of abscisic acid, epibrassinolide, polyethylene glycol, naphthaleneacetic acid, 6-benzylaminopurine and arabinogalactan proteins. Euphytica 195 (3):369-382. doi:10.1007/s10681-013-1001-4

40. Corral-Martínez P, Seguí-Simarro JM (2012) Efficient production of callus-derived doubled haploids through isolated microspore culture in eggplant (Solanum melongena L.). Euphytica 187 (1):47-61. doi:10.1007/s10681-012-0715-z

41. Custers JBM, Cordewener JHG, Fiers MA, Maassen BTH, van Lookeren-Campagne MM, Liu CM (2001) Androgenesis in Brassica: a model system to study the initiation of plant embryogenesis. In: Bhojwani SS, Soh WY (eds) Current trends in the embryology of angiosperm. Kluwer Academic Publishers, Dordrecht, The Netherlands, pp 451-470

42. San Noeum LH (1976) Haploides d'Hordeum vulgare L. par culture in vitro d'ovaries non fécondés. Ann Amélior Plantes 26:751-754

43. Fayos O, Vallés MP, Garcés-Claver A, Mallor C, Castillo AM (2015) Doubled haploid production from Spanish onion (Allium cepa L.) germplasm: embryogenesis induction, plant regeneration and chromosome doubling. Front Plant Sci 6. doi:10.3389/fpls.2015.00384

44. Bohanec B (2002) Doubled-haploid onions. In: Rabinowitch HD, Currah L (eds) Allium Crop Science: Recent Advances. CABI Publishing, pp 145-157

45. Li JW, Si SW, Cheng JY, Li JX, Liu JQ (2013) Thidiazuron and silver nitrate enhanced gynogenesis of unfertilized ovule cultures of Cucumis sativus. Biol Plant 57 (1):164-168. doi:10.1007/s10535-012-0269-x

46. Doi H, Hoshi N, Yamada E, Yokoi S, Nishihara M, Hikage T, Takahata Y (2013) Efficient haploid and doubled haploid production from unfertilized ovule culture of gentians (Gentiana spp.). Breed Sci 63 (4):400-406. doi:10.1270/jsbbs.63.400

47. Raquin C (1985) Induction of haploid plants by in vitro culture of Petunia ovaries pollinated with irradiated pollen. Z Pflanzenzüchtg 94 (2):166-169

48. Tang F, Tao, Y., Zhao, T., \& Wang, G. (2006) In vitro production of haploid and doubled haploid plants from pollinated ovaries of maize (Zea mays). Plant Cell Tissue Organ Cult 84 (2):233-237 
49. Kantartzi S, Roupakias D (2009) In vitro gynogenesis in cotton (Gossypium sp.). Plant Cell Tissue Organ Cult 96 (1):53-57. doi:10.1007/s11240-008-9459-9

50. Troung-Andre I (1998) In vitro haploid plants derived from pollination by irradiated pollen on cucumber. Paper presented at the Cucurbitaceae 88: Proceedings of the Ixth Eucarpia Meeting on Genetics and Breeding of Cucurbitaceae, France,

51. Germanà MA, Chiancone B (2001) Gynogenetic haploids of Citrus after in vitro pollination with triploid pollen grains. Plant Cell Tissue Organ Cult 66 (1):59-66

52. Bouvier L, Zhang Y-X, Lespinasse Y (1993) Two methods of haploidization in pear, Pyrus communis L.: greenhouse seedling selection and in situ parthenogenesis induced by irradiated pollen. Theor Appl Genet 87 (1-2):229-232

53. Zhang YX, Lespinasse Y (1991) Pollination with gamma-irradiated pollen and development of fruits, seeds and parthenogenetic plants in apple. Euphytica 54 (1):101-109. doi:10.1007/bf00145636

54. Hooghvorst I, Torrico O, Hooghvorst S, Nogués S (2020) In situ Parthenogenetic Doubled Haploid Production in Melon "Piel de Sapo" for Breeding Purposes. Front Plant Sci 11 (378). doi:10.3389/fpls.2020.00378

55. Kurtar ES, Balkaya A (2010) Production of in vitro haploid plants from in situ induced haploid embryos in winter squash (Cucurbita maxima Duchesne ex Lam.) via irradiated pollen. Plant Cell Tissue Organ Cult 102 (3):267-277. doi:10.1007/s11240-010-9729-1

56. Germanà MA (2006) Doubled haploid production in fruit crops. Plant Cell Tissue Organ Cult 86 (2):131-146

57. Ishii T, Karimi-Ashtiyani R, Houben A (2016) Haploidization via Chromosome Elimination: Means and Mechanisms. Ann Rev Plant Biol 67 (1):421-438. doi:10.1146/annurev-arplant-043014-114714

58. Kalinowska K, Chamas S, Unkel K, Demidov D, Lermontova I, Dresselhaus T, Kumlehn J, Dunemann F, Houben A (2019) State-of-the-art and novel developments of in vivo haploid technologies. TAG Theoretical and applied genetics 132 (3):593-605. doi:10.1007/s00122-018-3261-9

59. Ravi M, Chan SWL (2010) Haploid plants produced by centromere-mediated genome elimination. Nature 464 (7288):615-618. doi:10.1038/nature08842

60. Baum M, Lagudah ES, Appels R (1992) Wide Crosses in Cereals. 43:117-143

61. Comai L, Tan EH (2019) Haploid Induction and Genome Instability. Trends Genet 35 (11):791-803. doi:10.1016/j.tig.2019.07.005

62. Devaux P (2003) The Hordeum bulbosum (L.) method. In: Maluszynski M, Kasha KJ, Forster BP, Szarejko I (eds) Doubled haploid production in crop plants. A manual. Kluwer Academic Publishers, Dordretch, the Netherlands, pp 15-19

63. Lange W (1971) Crosses between Hordeum vulgare L. and H. bulbosum L. I. Production, morphology and meiosis of hybrids, haploids and dihaploids. Euphytica 20 (1):14-29. doi:10.1007/bf00146769

64. Kasha KJ, Kao KN (1970) High frequency haploid production in barley (Hordeum vulgare L.). Nature 225:874-876

65. Wedzony M, Forster BP, Zur I, Golemiec E, Szechynska-Hebda M, Dubas E, Gotebiowska G (2009) Progress in doubled haploid technology in higher plants. In: Touraev A, Forster BP, Jain SM (eds) Advances in haploid production in higher plants. Springer, Dordrecht, Netherlands, pp 1-33

66. Inagaki MN (2003) Doubled haploid production in wheat through wide hybridization. In: Maluszynski M, Kasha KJ, Forster BP, Szarejko I (eds) Doubled haploid production in crop plants: a manual. Dordrecht, pp 53-58

67. Wędzony M (2003) Protocol for doubled haploid production in hexaploid triticale (x Triticosecale Wittm.) by crosses with maize. In: Maluszynski M, Kasha KJ, Forster BP, 
Szarejko I (eds) Doubled haploid production in crop plants: a manual. Dordrecht, pp 135140

68. Berger F, Grini PE, Schnittger A (2006) Endosperm: an integrator of seed growth and development. Curr Opin Plant Biol 9 (6):664-670. doi:10.1016/j.pbi.2006.09.015

69. Marsollier A-C, Ingram G (2018) Getting physical: invasive growth events during plant development. Curr Opin Plant Biol 46:8-17. doi:10.1016/j.pbi.2018.06.002

70. Widiez T, Ingram GC, Gutierrez-Marcos J (2017) Embryo-Endosperm-Sporophyte Interaction in Maize Seeds. In: Maize Kernel Development. Boston, MA, pp 95-107

71. Murovec J, Bohanec B (2012) Haploids and doubled haploids in plant breeding. In: Abdurakhmonov I (ed) Plant Breeding. InTech, Rijeka, Croatia,

72. Gilles LM, Khaled A, Laffaire J-B, Chaignon S, Gendrot G, Laplaige J, Bergès H, Beydon G, Bayle V, Barret P, Comadran J, Martinant J-P, Rogowsky PM, Widiez T (2017) Loss of pollen-specific phospholipase NOT LIKE DAD triggers gynogenesis in maize. The EMBO Journal 36 (6):707-717. doi:10.15252/embj.201796603

73. Jacquier NMA, Gilles LM, Pyott DE, Martinant J-P, Rogowsky PM, Widiez T (2020) Puzzling out plant reproduction by haploid induction for innovations in plant breeding. Nature Plants in press:in press. doi:10.1038/s41477-020-0664-9

74. Schnable PS, Wise RP (1998) The molecular basis of cytoplasmic male sterility and fertility restoration. Trends Plant Sci 3 (5):175-180. doi:https://doi.org/10.1016/S13601385(98)01235-7

75. Chase CD, Gabay-Laughnan S (2004) Cytoplasmic Male Sterility and Fertility Restoration by Nuclear Genes. In: Daniell H, Chase C (eds) Molecular Biology and Biotechnology of Plant Organelles: Chloroplasts and Mitochondria. Springer Netherlands, Dordrecht, pp 593-621. doi:10.1007/978-1-4020-3166-3_22

76. Bohra A, Jha UC, Adhimoolam P, Bisht D, Singh NP (2016) Cytoplasmic male sterility (CMS) in hybrid breeding in field crops. Plant Cell Rep 35 (5):967-993. doi:10.1007/s00299-016-1949-3

77. Dong Y-Q, Zhao W-X, Li X-H, Liu X-C, Gao N-N, Huang J-H, Wang W-Y, Xu X-L, Tang Z-H (2016) Androgenesis, gynogenesis, and parthenogenesis haploids in cucurbit species. Plant Cell Rep 35:1991-2019. doi:10.1007/s00299-016-2018-7

78. Gilles LM, Martinant J-P, Rogowsky PM, Widiez T (2017) Haploid induction in plants. Curr Biol 27 (20):R1095-R1097

79. Maine MJD (2003) Potato haploid technologies. In: Maluszynski M, Kasha KJ, Forster BP, Szarejko I (eds) Doubled Haploid Production in Crop Plants: A Manual. Dordrecht, pp 241- 247

80. Yao L, Zhang Y, Liu C, Liu Y, Wang Y, Liang D, Liu J, Sahoo G, Kelliher T (2018) OsMATL mutation induces haploid seed formation in indica rice. Nature Plants 4:530 533. doi:10.1038/s41477-018-0193-y

81. Liu H, Wang K, Jia Z, Gong Q, Lin Z, Du L, Pei X, Ye X (2020) Efficient induction of haploid plants in wheat by editing of TaMTL using an optimized Agrobacterium-mediated CRISPR system. J Exp Bot 71 (4):1337-1349. doi:10.1093/jxb/erz529

82. Liu C, Zhong Y, Qi X, Chen M, Liu Z, Chen C, Tian X, Li J, Jiao Y, Wang D, Wang Y, Li M, Xin M, Liu W, Jin W, Chen S (2020) Extension of the in vivo haploid induction system from diploid maize to hexaploid wheat. Plant Biotechnol J 18 (2):316-318. doi:10.1111/pbi.13218

83. Hussain T, Franks C (2019) Discovery of Sorghum Haploid Induction System. In: Zhao ZY, Dahlberg J (eds) Sorghum: Methods and Protocols. Springer New York, New York, NY, pp 49-59. doi:10.1007/978-1-4939-9039-9_4

84. Kloiber- Maitz M, Wieckhorst S, Bolduan C, Ouzunova M (2018) Haploidisierung in sorghum. EP3366778A1, 
85. Kermicle JL (1969) Androgenesis conditioned by a mutation in maize. Science 166 (3911):1422-1424. doi:10.1126/science.166.3911.1422

86. Coe EH (1959) A Line of Maize with High Haploid Frequency. Amer Nat 93 (873):381382. doi:10.1086/282098

87. Rokka VM (2009) Potato haploids and breeding. In: Touraev A, Forster BP, Jain SM (eds) Advances in haploid production in higher plants. Springer, Dordrecht, The Netherlands, pp 199-208

88. Spooner DM, Ghislain M, Simon R, Jansky SH, Gavrilenko T (2014) Systematics, Diversity, Genetics, and Evolution of Wild and Cultivated Potatoes. Bot Rev 80 (4):283383. doi:10.1007/s12229-014-9146-y

89. Amundson KR, Ordoñez B, Santayana M, Tan EH, Henry IM, Mihovilovich E, Bonierbale M, Comai L (2020) Genomic Outcomes of Haploid Induction Crosses in Potato (Solanum tuberosum L.). Genetics 214 (2):369-380. doi:10.1534/genetics.119.302843

90. Britt AB, Kuppu S (2016) Cenh3: An Emerging Player in Haploid Induction Technology. Front Plant Sci 7:357. doi:10.3389/fpls.2016.00357

91. Jackson D (2017) No sex please, we're (in)breeding. The EMBO Journal 36 (6):703-704. doi:10.15252/embj.201796735

92. Geiger HH, Gordillo GA (2009) Doubled haploids in hybrid maize breeding. Maydica 54 (4):485-499 


\section{Figure legends}

Figure 1. Basic concepts concerning haploidy. A. Haploid generally refers to a cell (or organism) containing half the chromosome number found in somatic cells. However, according to the ploidy level of the considered species, a haploid individual may harbor one or several versions of each chromosome. Examples are shown for a theoretical diploid, tetraploid and hexaploid, each with a set of two different, non homologous chromosomes ( $\mathrm{x}=$ 2). B. Simple representation of the contrasted life cycles of bryophytes, left and angiosperms, right, using maize as example. Turquoise color refers to the gametophytic (haploid) stage, and dark red to the sporophytic (diploid) stage. Note that some organisms' life cycle relies mostly on the haploid gametophytic stage. On the other hand, angiosperms drastically reduced their haploid phase to a few cells embedded in the diploid tissue.

Figure 2. Overview of in vitro and in vivo haploidization methods in plants. Intersection depicts in vivo methods that necessitate in vitro step(s) to prevent haploid embryo abortion.

Figure 3. Different alternatives to produce male-derived DHs. Microsporogenesis and microgametogenesis are natural pathways that normally take place to give rise to the male gametophyte (pollen grain) and male gametes (sperm cells). Male-derived haploids or DHs may arise from deviations of these pathways at three different levels: (1) diverting the meiocyte, (before microspore release) towards proliferation to induce in vitro the formation of callus, from which haploids and DHs, but also heterozygous diploids, can be produced by organogenesis (Route 1); (2) reprogramming the vacuolate microspore or young pollen grain towards embryogenesis (or alternatively callus formation + regeneration through organogenesis) by the in vitro application of a stress treatment and subsequent in vitro culture (Route 2); and (3) in vivo elimination of the female genome after egg fertilization by a sperm cell (Route 3). See text for further details.

Figure 4. Different alternatives to produce female-derived haploids and DHs. Megasporogenesis and megagametogenesis are pathways that normally take place to give rise to the female gametophyte (embryo sac) and female gametes (egg cell and central cell). Femalederived haploids or DHs may arise by two main means: (1) Reprogramming of egg cell 
development into haploid embryogenesis, either by in vitro induction or in vivo pollination with haploid inducer lines (Route 4); and (2) the sperm cell fertilizes the egg cell, but the male genome is progressively eliminated during early embryogenesis. Some methods lead to viable seeds with haploid embryos and are thus fully in vivo, whereas other methods need additional steps of in vitro culture. 


\section{Diploid}

organism

Sporophyte

2n

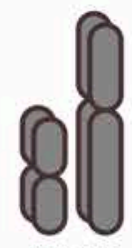

$2 n=2 x$

$\downarrow \hat{i}$

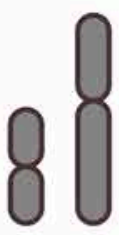

$\mathrm{n}=1 \mathrm{x}$
Tetraploid

organism

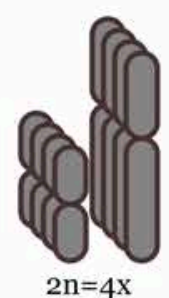

$\downarrow \hat{i}$

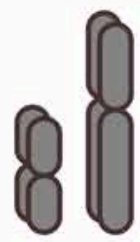

$\mathrm{n}=2 \mathrm{x}$
Hexaploid

organism

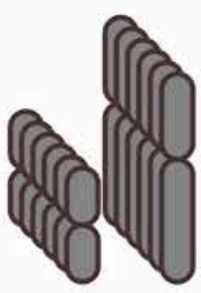

$2 n=6 x$

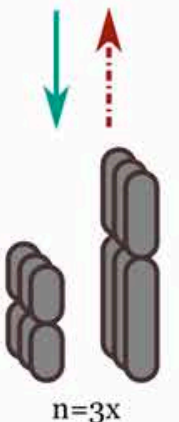

Haploidization: reduction by half of the chromosmoe set contained in the sporophyte. In the higher plant life cycle haploidization occurs naturally during meiosis. It is artificially triggered in DH technologies

Diploidization: Process through which total chromosome number is doubled. In the higher plant life cycle, A diploidization occurs naturally during fertilization. In DH technology, diploidization is usually triggered by cell cycle inhibitors such as colchicine, which permit replication but prevent cell division.

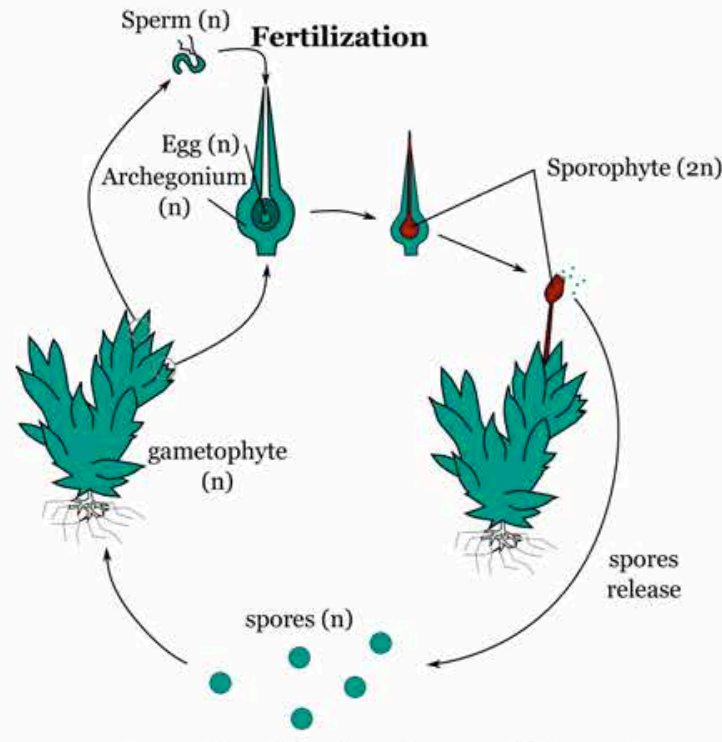

Bryophyte haplo-dominant life cycle

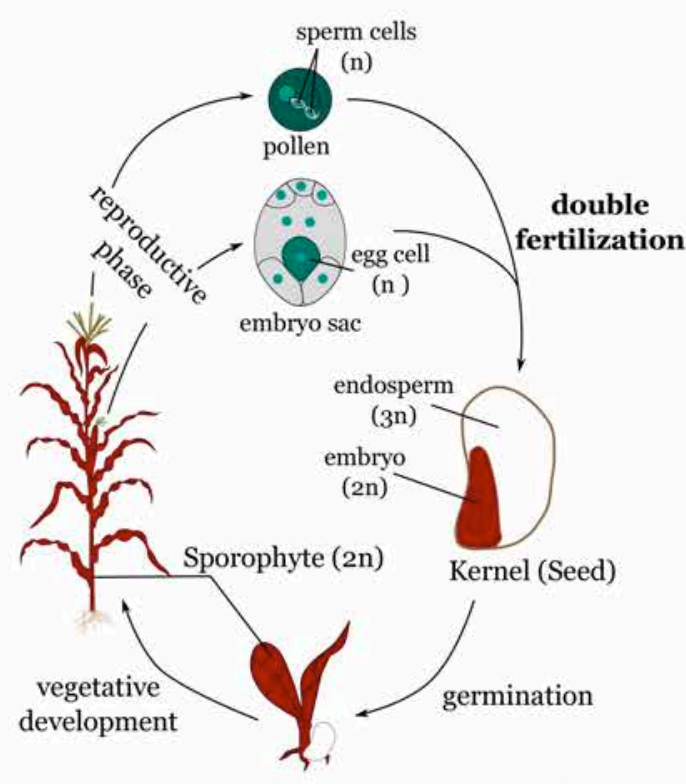

Angiosperms diplo-dominant life 


\section{In vitro methods}

In vitro culture of:

- microspores

- anthers

- ovaries

- ovules

- Flower buds
In vivo + in vitro methods

- Wide crosses

- Pollen treatments
In vivo methods

- Haploid inducer lines (including CENH3-based haploid inducer lines) 


\section{Megasporogenesis/ megagametogenesis}

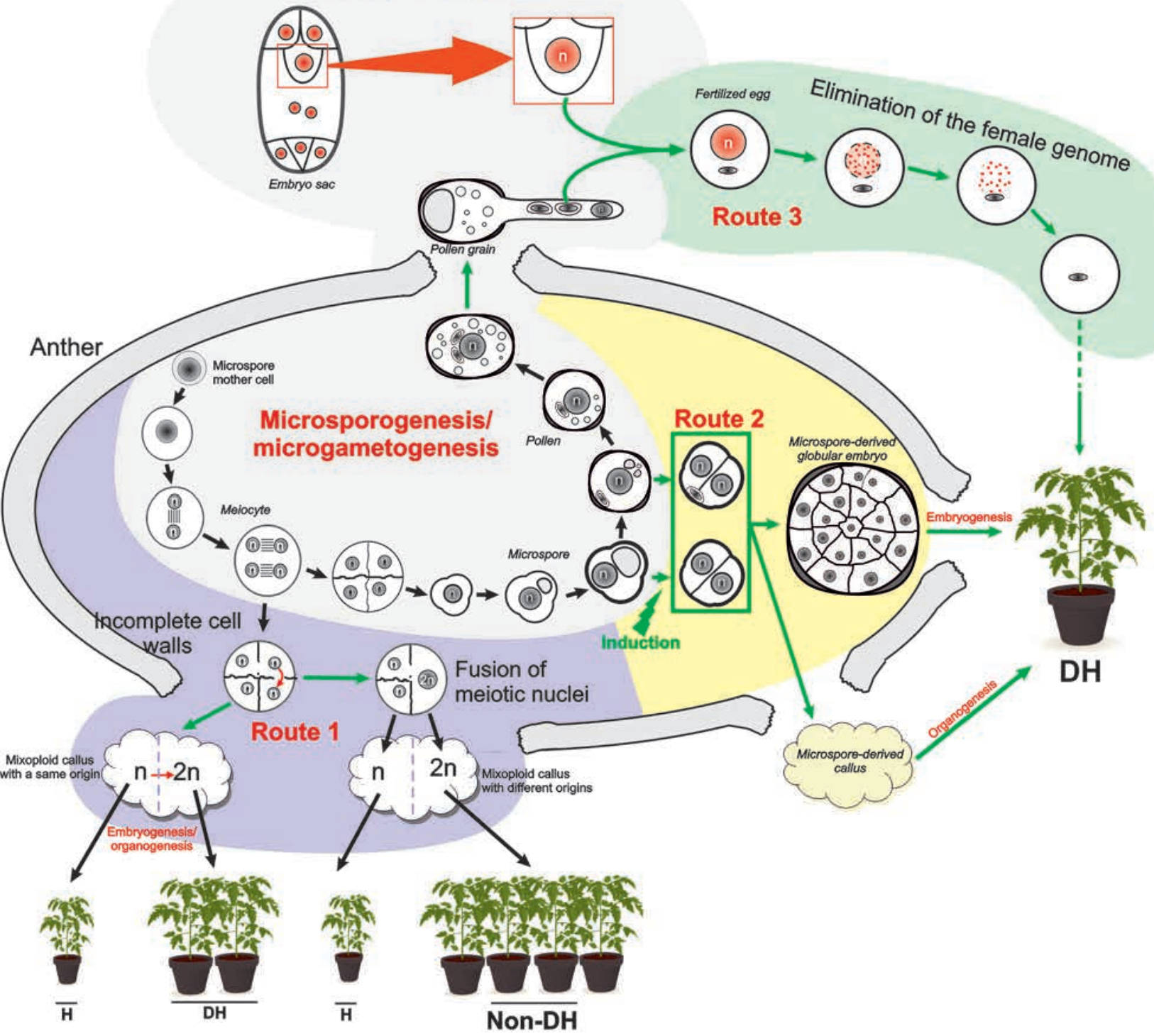




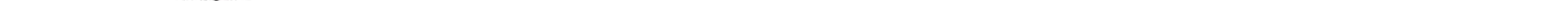

